\title{
VOLVULUS OF SIGMOID COLON IN A CHILD
}

\section{H. Budhichandra Singh ${ }^{1}$, M. Mahele ${ }^{2}$, Tozo Luwang ${ }^{3}$, Ch. Gyan Singh ${ }^{4}$, H. Manihar Singh ${ }^{5}$}

\section{HOW TO CITE THIS ARTICLE:}

H. Budhichandra Singh, M. Mahele, Tozo Luwang, Ch. Gyan Singh, H. Manihar Singh. "Volvulus of Sigmoid Colon in a Child". Journal of Evolution of Medical and Dental Sciences 2015; Vol. 4, Issue 34, April 27; Page: 59765980, DOI: $10.14260 /$ jemds/2015/870

ABSTRACT: Sigmoid volvulus although a common cause of large bowel obstruction in the elderly, is encountered rarely in the pediatric age group. We report a case of sigmoid volvulus in a 10-year-old child. The various predisposing factors for sigmoid volvulus in children are discussed, and the literature on the subject is also reviewed.

KEYWORDS: Sigmoid Volvulus, Paediatric.

INTRODUCTION: The term "volvulus" originates from Latin volvere, "to twist." Volvulus occurs when a segment of the alimentary tract twists on its mesenteric axis with a greater than 180-degree rotation, producing closed loop obstruction of intestinal lumen and mesenteric vessels, possibly leading to ischemia and gangrene. ${ }^{1}$

The most common sites of volvulus are the sigmoid colon and caecum. Volvulus of other portions of the alimentary tract, such as the stomach, gallbladder, small bowel, splenic flexure, and transverse colon are rare. ${ }^{2}$

Sigmoid volvulus is the most common large bowel volvulus in adults; in children, however, colonic volvulus is uncommon. Sigmoid volvulus is a disease of the elderly, often in those who are institutionalized and debilitated with neurologic and psychiatric conditions. The first report of Sigmoid volvulus in a 14-day-old boy was published in 1961 whereas the youngest patient reported is a 1-day-old boy with anal stenosis.3,4 Sigmoid volvulus is rarely considered in the differential diagnosis of abdominal pain (acute or recurrent) in neonates and children and this could be responsible for the devastating outcomes.

In elderly population, acquired redundancy of the sigmoid colon as a result of constipation is an important predisposing factor. ${ }^{5}$ However, this is not the case in the pediatric age group where sigmoid volvulus is rare, and in a large number of reported cases, a definite predisposing factor is present. This is a case report of sigmoid volvulus in a 10-year-old male child, treated at Regional Institute of Medical Sciences, Imphal, India, outlining aspects of diagnosis and management.

CASE REPORT: A 10-year-old male child without significant medical history presented to our hospital emergency department with absolute constipation for 2 days. The patient had received some symptomatic treatment with analgesics, proton pump inhibitors and sodium phosphate enema at a primary health care Centre without experiencing much improvement.

At the time of presentation to our institution, the patient had complaints of diffuse abdominal pain, distension, nausea, vomiting and fever. Eighteen hours prior to his presentation to the hospital he started to have sudden onset generalized abdominal pain, 3 episodes of bilious vomiting with progressively distension of the abdomen. He had no similar illness in the past.

On examination, the patient looked pale and toxic with temperature of $38.2^{\circ} \mathrm{C}$. The abdomen was markedly distended, tympanitic with rebound tenderness. The bowel sounds were absent. Per- 


\section{CASE REPORT}

rectal-examination showed a collapsed rectum with a small amount of normal color stool and one chewing gum was removed per rectally. Hernial orifices and external genitalia were intact.

Immediate resuscitation was done with intravenous fluids, broad spectrum antibiotics, nasogastric decompression etc. and monitoring of vital parameters. Ultrasound screening of the abdomen showed free fluid in the right iliac fossa, appendix was not visualized. Routine investigations for general fitness was done.

A provisional diagnosis of strangulated small bowel obstruction was made with differential diagnosis of perforated appendicitis in mind. The patient was immediately taken to the operating room for exploratory laparotomy and was found to have a 25 to $30 \mathrm{~cm}$ of dilated gangrenous sigmoid colon twisted $360^{\circ}$ clockwise on its mesentery. Pathological findings consisted of a long, redundant sigmoid loop rotated around an elongated mesentery with narrow parietal attachment.

Rest of the bowel and other viscera were normal. After derotation of the volvulus counterclockwise, the gangrenous sigmoid colon was resected and end to end anastomosis, after mobilizing descending colon, with rectum was done following on table bowel decompression and thorough lavage.

Intraoperatively, he received 1 unit of PRBC and another unit postoperatively. No intraoperative complications occured and the patient was transferred to the surgical intensive care unit. The patient had an uneventful postoperative course and was discharged on postoperative day 9. Histology of the resected colon showed necrotic colon with viable resection margins.

DISCUSSION: Sigmoid volvulus is encountered so rarely in paediatric practice its true incidence is not known. ${ }^{6}$ The median age is 7 years and there is a strong male predominance (Male: Female $=3.5: 1$ ) with a wide geographic variation. ${ }^{7}$ The presentation can range from acute to recurrent abdominal pain that is often relieved by passage of stool or flatus. The diagnosis is usually missed or delayed with devastating consequences and constipation is a common misdiagnosis.

Constipation is reported in 33\%-55\% of children with sigmoid volvulus but it is a sequelae not a predisposing factor for volvulus, and other factors play a role. ${ }^{8}$

In congenital anomalous fixation of the colon, sigmoid colon is long and redundant, mesocolon is long and narrow at its parietal attachments. ${ }^{9}$

Sarioglu et al ${ }^{10}$ reported 2 cases and reviewed 8 other cases from the literature of Hirschsprung's disease with colonic volvulus, 8 of them had sigmoid volvulus and interestingly all were males. These patients usually have short segment Hirschsprung's disease and if the mesosigmoid is freely mobile, the dilated ganglionic sigmoid colon twist forming a sigmoid volvulus.

Segmental dilatation of the colon is an abnormally dilated intestinal segment located between normally functioning proximal and distal bowel segments. Ravansse et al ${ }^{11}$ reported the first 2 cases of sigmoid volvulus as a complication of segmental dilation of the colon.

Other factors documented in literature are mental retardation, malrotation, Prune-belly syndrome, myotonic dystrophy. ${ }^{8}$

Our patient has congenitally long and redundant sigmoid colon and history of constipation with consumption of high fibre diet which contributed to the development of sigmoid volvulus.

A plain abdominal x-ray is suggestive of sigmoid volvulus in $29 \%$ of cases, while barium enema is diagnostic in $61 \%$ of cases. ${ }^{8}$ 
Barium enema is valuable not only in terms of diagnosis, but it may also relieve the obstruction by reducing the volvulus. However, this may require repeated enemas and is contraindicated in patients with suspected peritonitis.

Sigmoidosocopy is the initial treatment for those patients without peritoneal signs where the risk of perforation is high. Decompression rates vary from $70 \%$ to $90 \%$ success. Insertion of a rectal tube will further decompress the viable bowel. ${ }^{12}$

Expectant management is not recommended as spontaneous reduction occurs in only $2 \%$ of patients and recurrence is high in this group. ${ }^{13}$

The definitive treatment of sigmoid volvulus is surgical resection of the sigmoid colon and primary anastomosis. In case of gangrenous colon, resection and primary anastomosis may be performed if the patient is stable and if anatomic conditions are appropriate for a tension-free anastomosis. Hartmann's procedure or Mikulicz's colostomy are other alternatives. In nongangrenous cases, only derotation may be performed in high-risk with a volvulus-preventing procedure, like mesosigmoidoplasty, sigmoidopexy, or mesocoloplasty followed by bowel preparation and elective resection with end to end anastomosis. ${ }^{14,15}$

Simple derotation alone is not sufficient as this is associated with a high rate of recurrence. ${ }^{8}$

The overall mortality rate for SV is $6 \%$ while operative and neonatal mortality has been reported as $8.1 \%$ and $14 \%$, respectively. ${ }^{7}$ The most common cause of death in patients with a volvulus is sepsis. Other causes include pneumonia, intracranial hemorrhage, malnutrition, renal failure or hepatic failure, continued bowel obstruction and other life-threatening anomalies. Causes of long-term postoperative morbidity include adhesive bowel obstruction and recurrent volvulus.

CONCLUSION: This report describes sigmoid volvulus in an otherwise healthy young patient with delay in definitive diagnosis because of the unconventional patient profile. The case emphasizes the importance of early identification in the atypical patient before the appearance of twisted loop gangrene, in order to optimize patient management.

\section{REFERENCES:}

1. Gerwig WH. Volvulus of the colon: symposium on function and disease of anorectum and colon. Surg Clin North Am. 1955; 1395-9.

2. Hodin RA. Sigmoid volvulus. 2014.Available from URL: http://www.uptodate.com

3. Carter R, Hinshaw DB. Acute sigmoid volvulus in children. Am J Dis Child 1961; 101: 631-4.

4. De Caluwé D, Kelleher J, Corbally MT. Neonatal sigmoid volvulus: A complication of anal stenosis. J Pediatr Surg 2001; 36: 1079-81.

5. Kerry RL, Ranson HK. Volvulus of the Colon: Etiology, diagnosis and treatment. Arch Surg 1969; 99: 215-9.

12. Bruusgard C. Volvulus of the sigmoid colon and its treatment. Surgery 1947; 22: 466-78.

6. Patel U, Bhautik M. Choice of operative technique for emergency cases of sigmoid volvulus in a tertiary care hospital of Gujarat. NJMR 2012 Apr; 2(2): 226-8.

7. Salas S, Angel CA, Salas N, Murillo C, Swischuk L. Sigmoid volvulus in children and adolescents. J Am Coll Surg 2000; 190: 717-23.

8. Smith SD, Golladay ES, Wagner C, Seibert JJ. Sigmoid Volvulus in childhood. South Med J 1990; 83: 778-81. 


\section{CASE REPORT}

9. Khanna PR, Gangopadhyay AN, Shahoo SP, Khanna AK. Sigmoid volvulus in Childhood: Report of six cases. Pediatr Surg Int 2000; 16: 132-3.

10. Sorioglu A, Tanyel FC, Buyukpamukcu N, Hicsonmez A. Colonic Volvulus: A rare presentation of Hirschsprung's Disease. J Pediatr Surg 1997; 32: 117-8.

11. Ravasse PH, Petit TH, Cau D, Delmas P. Volvulus of the sigmoid colon as a complication of segmental dilatation of the colon. Eur J Pediatr Surg 1996; 6: 375-7.

12. Madiba TE, Thomson SR.The management of sigmoid volvulus. J R Coll Surg Edinb. 2000; 45: 74-80.

13. Reilly PMJ, Jones B, Bulkley GB. Volvulus of the colon. In: Cameron JL,ed. Current Surgical Therapy. St Louis, MO: Decker Inc; 1992.p.170-4.

14. Puneet, Khanna R, Gangopadhyay AN, Shahoo SP, Khanna AK. Sigmoid volvulus in childhood: Report of six cases. Pediatr Surg Int 2000; 16: 132-3.

15. Bhatnagar BN. Extraperitonealization of sigmoid for prevention of recurrence of volvulus - a follow-up study. Am J Proctol 1977; 28: 37-44.

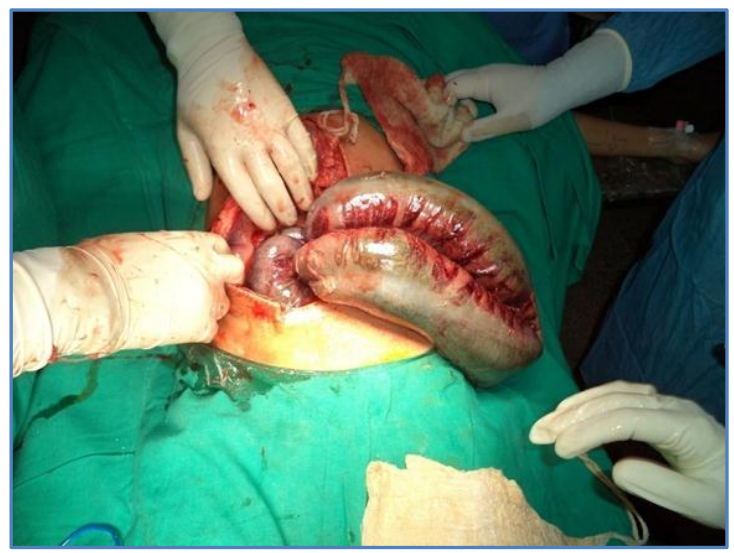

Fig. 1: Hugely distended gangrenous Sigmoid colon

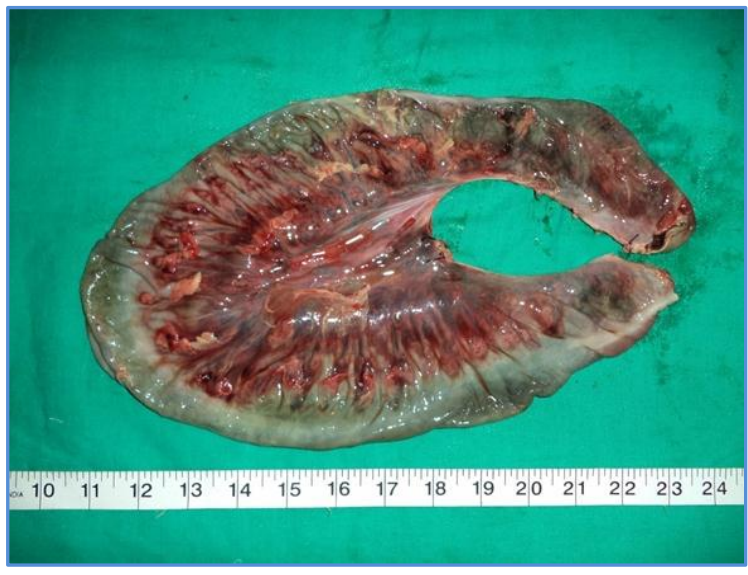

Fig. 2: Resected specimen of sigmoid colon 


\section{CASE REPORT}

\section{AUTHORS:}

1. H. Budhichandra Singh

2. M. Mahele

3. Tozo Luwang

4. Ch. Gyan Singh

5. H. Manihar Singh

\section{PARTICULARS OF CONTRIBUTORS:}

1. Resident, Department of General Surgery, Regional Institute of Medical Sciences, Imphal, Manipur.

2. Resident, Department of General Surgery, Regional Institute of Medical Sciences, Imphal, Manipur.

3. Resident, Department of General Surgery, Regional Institute of Medical Sciences, Imphal, Manipur.

\section{FINANCIAL OR OTHER}

COMPETING INTERESTS: None
4. Assistant Professor, Department of General Surgery, Regional Institute of Medical Sciences, Imphal, Manipur.

5. Professor, Department of General Surgery, Regional Institute of Medical Sciences, Imphal, Manipur.

\section{NAME ADDRESS EMAIL ID OF THE} CORRESPONDING AUTHOR:

Dr. H. Budhichandra Singh, Post Graduate Hostel No. 1, Room No. 27, RIMS, Imphal-795004.

E-mail: hbchandrasingh@gmail.com

Date of Submission: 13/02/2015.

Date of Peer Review: 14/02/2015.

Date of Acceptance: 17/04/2015.

Date of Publishing: 27/04/2015. 